\title{
Observational phenomena in the field of Kerr Superspinars
}

\author{
Zdeněk Stuchlík and Jan Schee \\ Institute of Physics, Faculty of Philosophy and Science, Silesian university in Opava, \\ CZ-74601. Opava, Czech Republic \\ email: zdenek.stuchlik@fpf.slu.cz, jan.schee@fpf.slu.cz
}

\begin{abstract}
We test observational consequences of primordial Kerr superspinars indicated by string theories. We demonstrate that Kerr superspinars can serve as ultra-high energy accelerators and explore specific optical phenomena related to accretion discs orbiting them.
\end{abstract}

Keywords. Kerr superspinar, Collisions, Optical phenomena

\section{Introduction}

Primordial Kerr superspinars are suggested by string theories, Gimon \& Hořava (2009). Such superspinars could survive up to the era of high redshift quasars, Stuchlík, Hledík \& Truparová (2011), demonstrating variety of unusual optical and astrophysical phenomena related to accretion discs orbiting them, Stuchlík \& Schee (2012).

\section{Kerr superspinars as a laboratory of ultra high energy physics}

For collision of a particle freely falling from infinity with a particle on a circular orbit at $r=1$ of a near-extreme Kerr superspinar with $a=1+\delta, \delta \ll 1$, the CM energy

$$
E_{C M}^{2} \sim 2 m^{2} \frac{1}{\sqrt{2 \delta}}
$$

In the case of head-on collisions of particles freely falling from infinity along radial trajectories having $\theta=$ const, with particles of the same kind that inverted their motion near $r=0$, Stuchlík (1980), we find possibility of much higher efficiency in obtaining extremely large CM energy due to the approximate relation, Stuchlík \& Schee (2012),

$$
E_{C M}^{2} \sim 2 m^{2} \frac{1}{\left[\delta\left(1+\sin ^{2} \theta\right)\right]} .
$$

We see that in the field of near-extreme superspinars, when $\delta$ can be assumed very small, e.g., $\delta \sim 10^{-12}$, or smaller, the extremely-high energy processes can frequently occur, with energy amplification of order $\sim 10^{6}$ or higher. We expect that generated particles are highly relativistic (or high-frequency photons). The created particles (photons) are distributed isotropically in the CM system. For collisions of the radially moving particles the CM system is identical with the LNRF. The LNRF photon escape cones, Stuchlík \& Schee (2010), give space and energy distribution of escaping photons as illustrated in Fig. 1. The frequency shift relative to distant static observer is given by

$$
g=\frac{\left[1-\frac{2 r}{\Sigma}\left(1-a \Omega \sin ^{2} \theta\right)^{2}-\left(r^{2}+a^{2}\right) \Omega^{2} \sin ^{2} \theta\right]^{1 / 2}}{1-\lambda \Omega},
$$



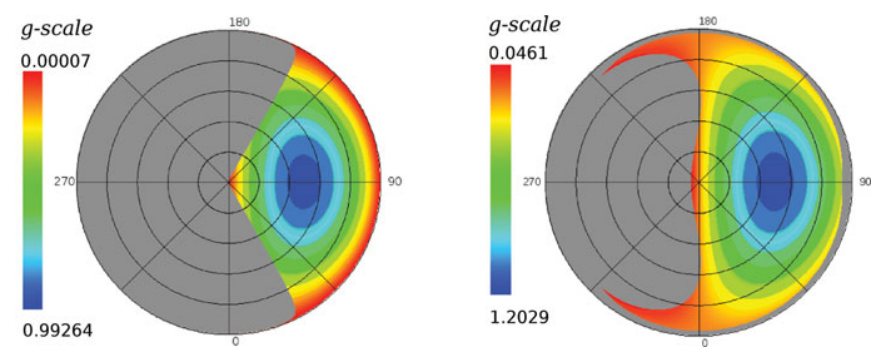

Figure 1. Escape cones for two represenative values of superspinar spin $a=1+10^{-7}$ (left) and $1+8 \times 10^{-2}$ (right) and inclination angle $\theta=85^{\circ}$.

where is $\Omega=\Omega_{L N R F}=2 a r /\left[\left(r^{2}+a^{2}\right)^{2}-\Delta \sin ^{2} \theta a^{2}\right]$, and $\lambda$ is the axial impact parameter of the photon.

\section{Profiled Lines from Keplerian Disc}

Radiating Keplerian disc is composed of locally monochromatic and isotropically radiating point sources uniformly distributed along circular geodesics. The frequency shift $g$ and focusing $\mathrm{d} \Pi$ can then be determined enabling to compute the profiled spectral lines of radiation from the Keplerian disc, Schee \& Stuchlík (2009).
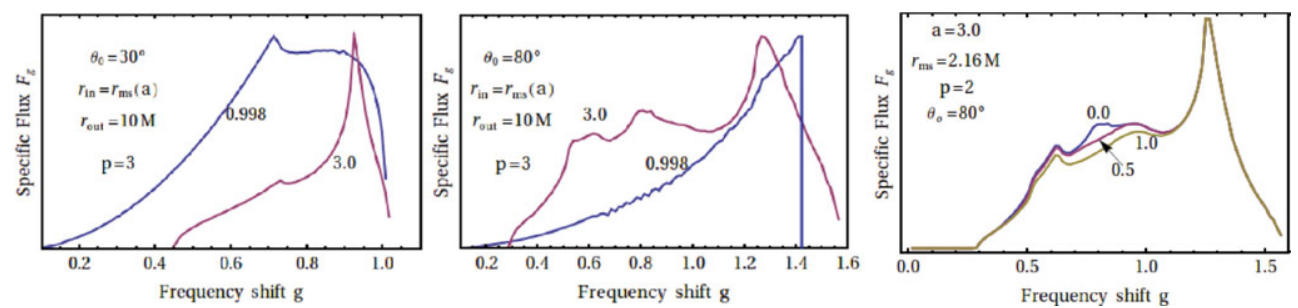

Figure 2. The profiled lines of the radiation from Keplerian discs in the vicinity of Kerr black hole and superspinar with spin $a=0.998$ (blue) and $a=3.0$ (red). The inner edge is at $r=r_{m s}$ and the outer edge is at $r=10 \mathrm{M}$. The inclination of the observer is $\theta_{o}=30^{\circ}$ (left) and $80^{\circ}$ (middle). Superspinar radius is $R_{s u s p}=0.1 \mathrm{M}$. The influence of superspinar radius is illustrated in plots on ther right. Emissivity law has standard form.

In Fig. 2 we demonstrate strong qualitative and quantitative differences between profiled lines created in the field of Kerr black holes and Kerr superspinars.

\section{Acknowledgements}

Supported by the project CZ.1.07/2.3.00/20.0071 Synergy.

\section{References}

Gimon, E. G. \& Hořava, P. 2009, Phys. Lett. B, 672, 299

Stuchlík, Z. 1980, Bull. of the Astronom. Inst. of Czechoslovakia, 31, 129

Schee, J. \& Stuchlík, Z. 2009, General Relativity and Gravitation, 41, 1795

Stuchlík, Z. \& Schee, J. 2010, Class. and Quant. Grav., 27, 215017

Stuchlík, Z., Hledík, S., \& Truparová, K. 2011, Class. and Quant. Grav., 28, 155017

Stuchlík, Z. \& Schee, J. 2012, Class. and Quant. Grav., 29, 065002 